\title{
Filament Chirality: A Link Between Fine-Scale and Global Patterns
}

\author{
Sara F. Martin ${ }^{1}$ \\ Helio Research, 5212 Maryland Ave., La Crescenta, CA 91214, USA
}

\begin{abstract}
Solar features that exhibit chirality are: fibril patterns in filament channels, filaments, coronal arcades over filaments, superpenumbral fibrils exterior to sunspots, whole active regions observed with magnetographs, some large-scale X-ray structures, and interplanetary magnetic plasma clouds originating from coronal mass ejections. Their signatures of chirality are briefly reviewed; some details are mentioned to further show relationships which link all of the chirality patterns into a single framework. The helicity of solar magnetic fields is the evident physical phenomena which links each of the seven features to each other and into a broader framework. The chirality system, viewed as a whole magnetic system, reveals: (1) a consistent rotational configuration of the dominant direction of the magnetic field with height of features within and over filament channels from the photosphere to the highest part of the solar corona, and (2) the prominence cavity as a unique space between magnetic fields of opposite helicity.
\end{abstract}

\section{Introduction}

Seven patterns of chirality have been found among solar features and are reviewed by Zirker et al. (1997). In the present paper, the seven patterns are revisited to clarify details and to amplify on the relationships between the patterns. The solar features, pictorially summarized in Figures 1 and 2, are: (1) fibrils in filament channels, (2) filaments, (3) systems of coronal loops overlying filaments, (4) super penumbral fibrils exterior to sunspots, (5) active regions as a whole, (6) large-scale X-ray structures, and (7) interplanetary clouds arising from coronal mass ejections which are often accompanied by erupting filaments.

The schematic diagrams in Figures 1 and 2 emphasize the chirality relationships among these solar features. A clear distinction needs to be made between two broad categories of patterns: (1) pairs of one-to-one relationships between each of the seven types of features, and (2) the statistical dominance of one of the two sets of invariant chirality relationships in the northern solar hemisphere and the opposite set in the southern hemisphere. The chirality pairs are represented in rows and the invariant relationships are contained within each column in Figures 1 and 2. The sets of relationships that are dominant in the northern and southern hemispheres are shown respectively in the left and right columns.

${ }^{1}$ Also at: California State University, Northridge, CA, USA 


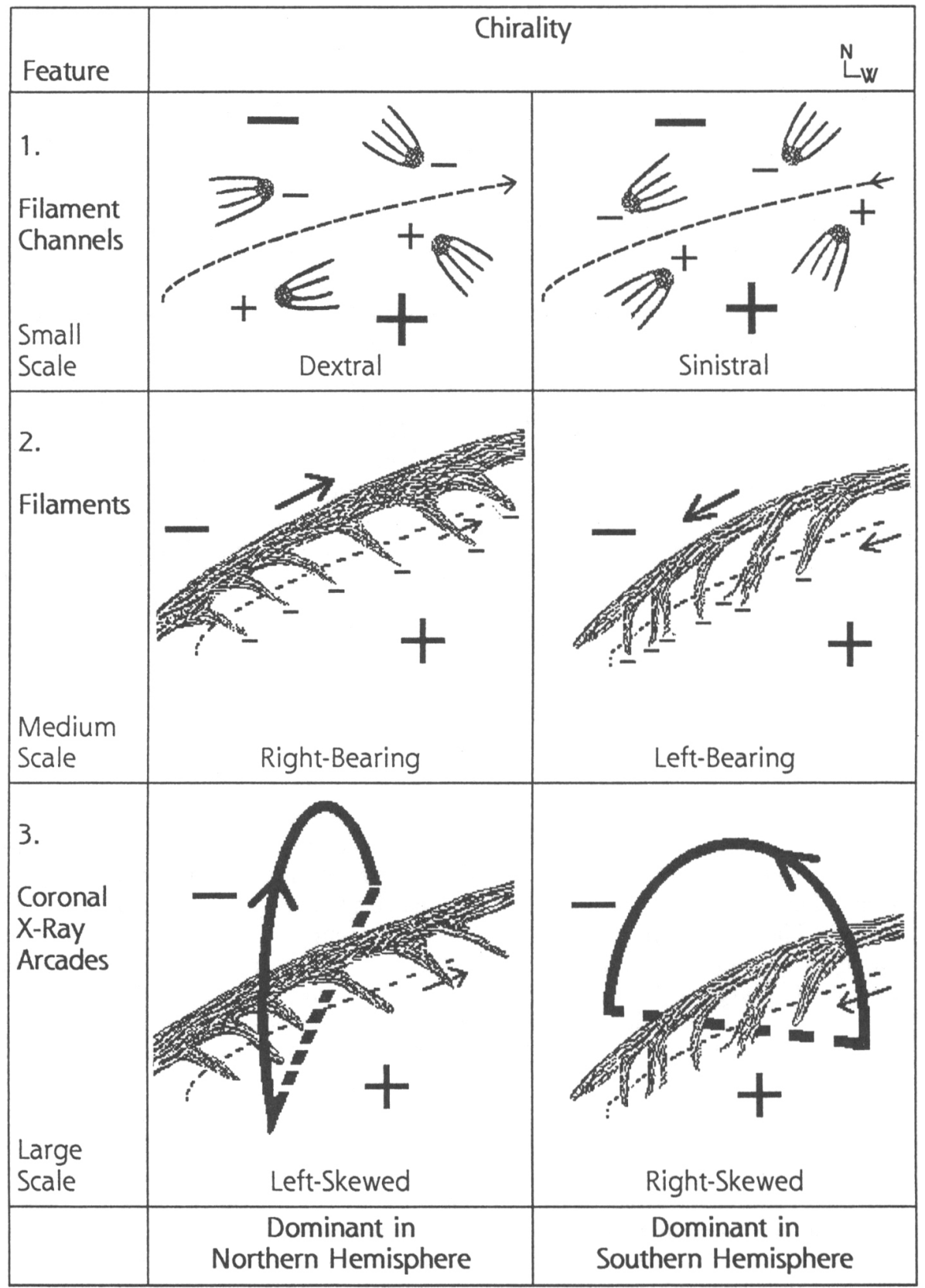

Figure 1. Chirality patterns of solar features involving filaments. 


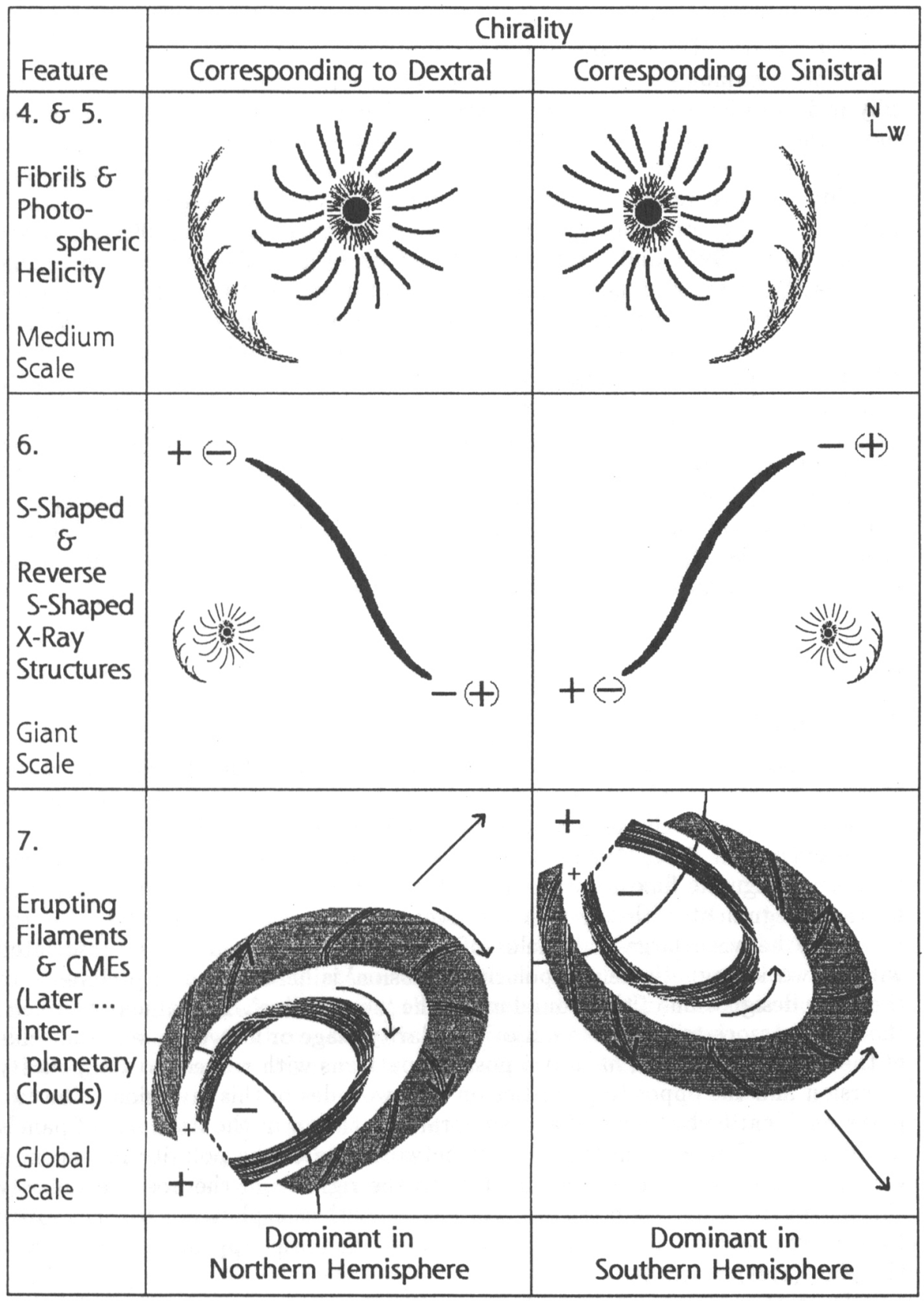

Figure 2. Other chirality patterns of solar features. 
Figure 2 is a continuation of Figure 1. Some data sets have revealed as many as one exception to the global hemispheric pattern per 3 chirality pairs while other sets of data have only included a few percent of exceptions to this hemispheric bias (Zirker, Leroy and Gaizauskas, 1998, these proceedings). The exceptions are not illustrated in Figures 1 and 2.

The invariant relationships within each column in Figures 1 and 2 imply physical links between these solar features. The obvious candidate for this linkage is the magnetic fields of these features. Magnetic fields exhibit chirality and are known to be basic to all of these solar features. However, the matching of chirality pairs is fraught with potential for differing interpretations of chirality in terms of magnetic helicity as presented by Rust and Kumar (1994), Low and Hundhausen (1995), and Martin and McAllister (1997). However, all agree that the helicity of solar magnetic fields is an important tool for achieving greater understanding of the physics of all seven features. Together, the chirality of these features offers a new opportunity to gain insight into changes in the solar atmosphere over the solar cycle. Does chirality, interpreted in terms of the helicity of solar magnetic fields, offer new information on the origin of the solar cycle? This possibility has been proposed (Rust and Kumar 1994) and is being questioned (Zirker et al 1997, Foukal 1998, these proceedings). At present, any tentative answer rests on interpretations, which in turn rest on assumptions and other interpretations. These, in turn, relate to the observations of chirality. Below we review the chirality pattern of each solar feature and mention a few salient details which must fit within the grand scheme.

\section{Chirality Patterns}

\subsection{Fibril Patterns}

The smallest solar features showing chirality are fibrils; but, fibrils only reveal chirality in relationship to other fibrils rooted in plage or plagettes of opposite magnetic field. The chirality patterns of fibrils are most clearly seen in filament channels (Foukal 1971, Martin et al. 1994). In channels of medium to high photospheric magnetic flux density, the central or key fibrils delineate the channel by their alignment with the polarity inversion. The 'polarity inversion' is the boundary between large-scale fields of opposite vertical component associated with network magnetic fields; 'polarity inversion' is herein used in preference to the older designation of this boundary as the 'neutral line'. In filament channels, the fibrils, associated with the majority polarity plage or network magnetic fields of the majority polarity, have two possible patterns with respect to the polarity inversion and the opposite polarities on the two sides of this inversion. The two patterns, identified as dextral and sinistral, are shown in the first pair of panels in Figure 1. Viewed from the positive network side of the polarity inversion, a channel is dextral if the fibrils emanate to the right from the positive polarity chromospheric plage or plagettes (associated with the photospheric network); from the same view, the channel is sinistral if the fibrils appear to stream from the plage(ttes) to the left.

Dextral and sinistral channels have mirror symmetry relative to each other. In both cases, the polarity boundary, between the oppositely directed fibrils, is not crossed by any single fibrils. This boundary corresponds to a locus of 
points where the magnetic field is nearly horizontal at the photosphere and nearly aligned with the polarity inversion. Typical examples are shown in active regions by Hagyard et al. $(1984,1990)$ and in a decaying active region by Zhang (1990).

The direction of the fibrils with respect to the dominant line-of-sight polarity indicates that the magnetic field of fibrils on both sides of the polarity boundary have a common component parallel to it, as first illustrated by Foukal (1971). Martin et al. (1994) schematically illustrate the inferred magnetic field geometry in a volume cross-section across a filament channel.

As shown by Filipov (1998, these proceedings), the fibrils in many filament channels have a strong tendency to be 'inverse' in the same sense that the magnetic fields in quiescent filaments are 'inverse' (Leroy 1978, 1989; Bommier and Leroy 1998, these proceedings). The inverse component of fibril patterns is not necessarily symmetric with respect to the polarity inversion but the pattern is such that the positive magnetic field has a component away from the polarity inversion and the negative component toward it. This pattern does not include local irregularities due to the development and decay of small bipoles known as ephemeral active regions.

\subsection{Filament Patterns}

As indicated in the middle pair of schematic diagrams in Figure 1, the chirality of filaments can be revealed by: (1) direct measures of the magnetic field along the axis of a filament relative to the polarity on the two sides of the filament, and (2) the direction of the barbs of filaments relative to filament axis along the polarity inversion, as best seen to date in $\mathrm{H} \alpha$ filtergrams. The chirality of the axial magnetic field is defined when viewing filaments from the positive network magnetic field side as in Figure 1. If its axial magnetic field points to the right, a filament is dextral; if the axial field component is toward the left, the filament is sinistral.

The chirality of the barbs can be defined independently of viewing angle or knowledge of any magnetic field polarity. Viewed from either side of the filament, if the barbs on the near side veer from the filament axis to the right and downward to the chromosphere, the barbs are right-bearing; if on the near side, they veer from the axis to the left and downward to the chromosphere, they are left-bearing. For filaments intermediate to active region filaments and quiescent filaments as illustrated in Figure 1, the barbs tend to be broader near the filament axis and converge to chromospheric points or clusters of chromospheric points. Mass motions can be either up or down along the barbs (Engvold 1998, these proceedings).

Martin and Echols (1994) and Martin (1994) identified the chromospheric ends of filament barbs with pockets of photospheric magnetic fields of the minority polarity along the sides of filaments. This association is consistent with the inverse magnetic fields in filaments as measured by Leroy and colleagues cited above, although these authors could not distnguish at that time between axial fields along the filament spine and barb fields extending away from the spine. In quiescent filaments, the barbs tend to dominate the structure; they are typically the brightest parts of quiescent prominences and much more likely to be measured than the axial component. If the barbs are measured at the limb 
instead of the axial component, the magnetic field would have to be 'inverse' to be consistent with their being rooted in small pockets of the minority polarity as schematically represented in Figure 1 . The barbs are clearly identified in prominence observations against the solar disk (Martin and Echols, 1995) and, in multi-day observations, large barbs can be traced to the "legs" of prominences when observed above the limbs such as in Figure 6 in Martin, Bilimoria and Tracadas (1994).

\subsection{The Skew of Coronal Arcades Overlying Filaments}

Martin and McAllister $(1995,1997)$ studied the skew of coronal arcades seen in $\mathrm{X}$-ray images from the Yohkoh satellite. Skew was defined as the acute angle between the loops and the polarity inversion or filament axis. If the arcade loops cross over the filament or filament channel in the sense of threads of a left-hand screw or right hand screw, they defined the loops as left-skewed or right-skewed, respectively. They investigated the skew of the systems of nearly parallel loops that comprise coronal arcades overlying filaments. As depicted in the third pair of panels in Figure 1, they found a one-to-one relationship between the skew of the arcades and the type of filament. The chirality relationship is in the inverse sense. Left-skewed arcades overlie dextral filaments and filament channels; right-skewed arcades overlie sinistral filaments and filament channels.

The chirality relationship applied to both quiescent arcades and dynamic arcades that evolve after filament eruption/coronal mass ejection events. Many of the dynamic arcades evolved such that the later-forming loops showed apparent rotation relative to the earlier-forming loops. This apparent rotation also has a specific sense. Left-skewed arcades revealed apparent rotation in the counterclockwise sense and right-skewed arcades in the clockwise sense. This is invariably in the direction of decreasing skew and decreasing magnetic shear with time and height of the loops as found previously by other authors. For example, Rust and Bar (1973) discuss post-flare loops, which in their successive formation, collectively showed apparent rotation from a $20^{\circ}$ angle to a $90-100^{\circ}$ angle with respect to the polarity inversion.

\subsection{Chirality of Superpenumbral Fibrils Around Sunspots}

Some of the longer, more conspicuous fibrils in active regions are those in an extended area exterior to the penumbra of sunspots. During the early part of the 20th century, these fibrils caught the attention of solar astronomers (Hale 1925,1927 ), who recognized the similarity between the fibril patterns and the pattern of iron filings around bar magnets. Richardson (1941) made an extensive study of fibril patterns around large sunspots over parts of 3 solar cycles from $\mathrm{H} \alpha$ spectroheliograms obtained daily at Mt. Wilson Observatory. For $1 / 3$ of these cases, he could identify the fibril pattern as being dominantly clockwise or counterclockwise around a portion of the perimeter of the penumbra. He also found some cases where both senses could be seen around different parts of the perimeter of the same sunspot. Nevertheless, he found, for superpenumbral fibrils with a dominant sense of chirality, that the majority with a counterclockwise pattern were in the northern hemisphere and that a majority with a clockwise pattern were in the southern hemisphere. Counterclockwise and clockwise are defined in following the curvature of the fibrils from the end of the fibril more 
distant from the penumbra to the end closest to the penumbra (first pair of panels in Figure 2).

Rust (1994) was the first to point out the consistency of the patterns of chirality in sunspots, filaments, active regions and magnetic clouds. In $1994 \mathrm{D}$. Rust asked me to check out his prediction of a close association between the chirality of superpenumbral fibril patterns and the chirality of nearby filaments. He anticipated that counterclockwise fibril patterns would be adjacent to dextral filaments and clockwise fibrils would be adjacent to sinistral filaments. I chose the first 10 sunspot pictures with clearly curved fibril patterns that could be found in a local file of photographic prints of high quality images from the Big Bear Solar Observatory. The filaments were either seen in the same prints or in full-disk $\mathrm{H} \alpha$ images taken on the same day. Rust's prediction was correct in all 10 cases (Rust and Martin 1994). This relationship is also illustrated in the first pair of panels in Figure 2. Typically I have observed that the superpenumbral fibrils show the most curvature on the side of the sunspot toward a filament or filament channel.

\subsection{The Chirality of Active Regions}

Extensive, smoothly curved patterns of fibrils are characteristic of the $\mathrm{H} \alpha$ chromosphere of active regions. Thus, it could be expected that active regions as a whole might exhibit chirality patterns similar to those in filament channels and around sunspots. Seehafer (1990) estimated the electric current helicity in 16 active regions and concluded that the electric current helicity is predominantly negative in the northern hemisphere and positive in the southern hemisphere. Pevtsov, Canfield and Metcalf (1994) also demonstrated the existence of chirality in active regions by analyzing vector magnetograms from the Mees Solar Observatory. Like superpenumbral fibrils, the whole active region does not have to be the same chirality for there to be a dominance of one pattern. Pevtsov, Canfield and Metcalf defined such a pattern using the deviation of vector magnetograph measurements from a potential magnetic field configuration. A dominant sense of chirality was found for most active regions. It is consistent with the chirality of the superpenumbral fibril patterns and Seehafer's estimate of current helicity, as previously pointed out by Rust (1994).

\subsection{The Chirality of Large-scale $X$-ray Structures}

Very large scale X-ray structures have been noticed to exhibit a slight $\mathrm{S}$ or backwards-S shape as a function of hemisphere (Harvey 1994, Rust and Kumar 1996). Harvey describes the pattern as a systematic twist around magnetic concentrations in both hemispheres which is consistent with differential rotation. The pattern, as viewed from above, is illustrated in the middle pair of panels in Table II. Overall, there is a dominance of reverse S-shaped structures in the northern hemisphere and S-shaped structures in the southern hemisphere.

The reverse-S and S-shaped patterns also apply to many filament channels and sets of X-ray structures closely aligned with filament channels (McAllister et al. 1998, these proceedings). These patterns apply mostly to features in the environment of "diagonal" filaments or filament channels forming within or between the decayed remnants of active regions. The "diagonal" form refers to the mid-section of filaments whose orientation is consistent with differential 
rotation acting on initial north-south oriented filament channels. Initial N-S oriented structures aligned or nearly aligned with the polarity inversion of the filament channel evolve to become NE-SW in the northern hemisphere and initial S-N oriented structures become SE-NW in the southern hemisphere. For these polarity inversions, the reverse S-shape in the northern hemisphere and $S$-shape in the southern hemisphere are as expected if the magnetic ends of these structures are rooted in the polarity of network fields which are consistent with the extreme ends of the dextral and sinistral axial fields of filaments and filament channels.

\subsection{The Chirality of Interplanetary Clouds, CMEs and Erupting Filaments}

Interplanetary clouds are the ejected parts of coronal mass ejections (CMEs), as first concluded by Marubashi $(1986,1997)$ and amplified by others. Many such plasma clouds have helical structure detected by magnetometers on board spacecraft (Burlaga 1991, Klein and Burlaga 1982). Gosling (1990) and Gosling and Birn (1995) proposed that helical magnetic fields in interplanetary clouds originate from magnetic reconnection in ascending coronal arcades. Based on the same scenario, Martin and McAllister $(1997,1998)$ anticipate that leftskewed coronal arcades become left-helical CMEs and later are detectable as left-helical interplanetary clouds; similarly right-skewed coronal arcades should become right-helical CMEs and interplanetary clouds. Consistent with this picture, Rust (1994) found that the majority of interplanetary clouds with lefthelical structure come from the northern solar hemisphere and most of those with right-helical structure originate in the southern hemisphere. The outer structure in the lower pair of panels in Figure 2 represent CMEs in each hemisphere which later become interplanetary clouds. The large-scale helical structures of the plasma clouds are consistent with the chirality of CMEs, rather than the chirality of filaments, confirming Marubashi's original association of interplanetary flux ropes with the coronal magnetic fields around filaments.

As represented by the inner twisted structures in the last pair of panels in Figure 2, Martin and McAllister $(1997,1998)$ have also predicted that dextral filaments develop right-helical twist via magnetic reconnection occurring in the pre-eruptive or early eruptive stage and sinistral filaments develop left-helical twist. Their hypothesis is based on a proposed model of a filament composed of an initially untwisted axial magnetic field aligned with the polarity inversion and barb magnetic fields which extend from the axial field of the filament spine to the chromosphere adjacent to the filament (Martin and Echols, 1994). Except for filaments in areas of low magnetic flux density, mass continuously streams along the spine and barbs, providing evidence of the magnetic fields parallel with the fine structures of the spine and barbs. During the early phase of eruption, the axial magnetic field ascends beneath the rising coronal arcade as observed in many coronal mass ejections. In this model, the magnetic fields of the filament begin to detach from the Sun by magnetic reconnection between barbs with oppositely directed magnetic fields beneath the rising axis of the erupting filament. The expectation of Martin and McAllister is that filament mass, if later identifiable within a plasma cloud, will have helicity opposite in sign to the encasing plasma from the CME. The prediction is more readily tested 
as erupting filaments leave the Sun; often helical structure is then quite readily seen (Rompolt 1975, 1990). For the filament only, the Martin and McAllister prediction gives the opposite sign of helicity from the Rust model (1994) and its continuation by Rust and Kumar (1994); the latter authors assume that the filament lies in the lower portion of a larger-scale helical magnetic field which supports the filament mass against gravity. Martin and McAllister make no assumption about the support of the filament mass because the structure of barbs is seen to have large vertical components, mass motions of several $\mathrm{km} / \mathrm{s}$, and no evidence of magnetic wells to support mass.

\section{Discussion}

Figures 1 and 2 present solar features exhibiting chirality from small scales to global scales. The chirality of filament channels, filaments and their overlying coronal arcades are all defined relative to the polarity inversion. The axial fields of quiescent filaments always maintain the chirality of the filament channel where their barbs are rooted. All arcades and features above the arcades exhibit the opposite sense of chirality. Between the filament and the adjacent and overlying coronal arcade is a region, void of emission, called "the cavity". There is no source of empirical information on the cavity magnetic field. Also, there is no requirement to assume that these magnetic fields are necessarily directly connected in the corona. It has already been shown (Figure 1) that the filament and surrounding arcade are rooted in separate magnetic fields of opposite polarity. Hence, it is plausible that the filament magnetic fields and coronal arcade fields are divided by a separatrix surface.

The chirality patterns thus reveal a new significance for the space called "the cavity"; it is the volume between features of opposite chirality. To better understand the significance of the cavity, it can be compared with the characteristics of other volumes of space where features of opposite chirality are juxtaposed. Martin, Bilimoria and Tracadas (1994) found that filaments of opposite chirality do not join. Instead they avoid each other by forming a cusp and this configuration allows the ends of filaments to be juxtaposed but not directly connected. Similarly, Pevtsov and Canfield (1997) have found that, near the equator, active regions of opposite chirality do not have interconnecting coronal loops while those of the same chirality have many interconnecting loops.

Above and below the coronal cavity, we have thus found the third example of solar features of opposite chirality which do not show evidence of being magnetically connected or a tendency to reconnect with each other in their quiescent state; filaments of one chirality are spatially isolated from the coronal loops of opposite chirality above. Even during filament eruptions and CMEs near the Sun, prominence mass remains isolated although encased within the larger CME. Thus, I propose that the existence of a prominence cavity is physically related to its location between features of opposite chirality.

The chirality of solar features can all be interpreted as features with magnetic helicity. Berger (1998, these proceedings) distinguishes between two forms of helicity, linking and twist. These forms of helicity can be increased, decreased or interchanged by magnetic reconnection. Prior to eruption, neither filaments nor coronal arcades exhibit large-scale twist in their fine structure, although the 
spatial resolution of many observations is adequate for seeing twist. However, during eruption, filaments often display large-scale twist. Likewise, interplanetary clouds, associated with erupted coronal arcades, exhibit large-scale helical structure. Thus in the pre-eruptive state, the chirality of filaments and coronal arcades can be interpreted either in terms of linking helicity or an absence of twist helicity above the photosphere. In their eruptive and post-eruptive state, their chirality is readily interpreted as twist helicity converted from the prior form of helicity by magnetic reconnection during the eruptive processes.

The nature of the chirality of solar features, and their interpretation in terms of helicity, and of the coronal cavity should become better understood with further study.

Acknowledgments. The material and concepts developed herein were assembled via support from: NASA grant NAGW-4300, its continuation under grant NAG5-4180, and a subcontract via JPL to California State University, Northridge for studies of the helicity of solar features with J. Feynman, A. Ruzmaikin and P. Liewer encouraged by G. Chapman. The initial work on the skew of coronal arcades was supported by P. Gilman, B.C. Low and A. Hundhausen while the author was a visitor at the High Altitude Observatory during 1994 and 1995. The author is indebted to colleagues O. Engvold, T. Forbes, V. Gaizauskas, K. Harvey, E. Priest, A. van Ballegooijen, and J. Zirker for extensive interactive discussions made possible by NSF grant ATM-9596070 to the Solar Physics Research Corporation and its continuation under grant ATM-9696256 to Helio Research. Beneficial discussions with C. Zwaan were made possible through NATO Collaborative Research Grant 910997.

\section{References}

Burlaga, L.F. 1991, in Physics of the Inner Heliosphere II, (eds.) R. Schwenn and E. Marsch, Springer Verlag, New York, p. 1

Foukal, P. 1971, Solar Phys., 19, 59

Gosling, J.T. 1990, in Physics of Magnetic Flux Ropes, (eds.) C.T. Russell, E.R. Priest, and L.C. Lee, Geophys. Mono. Ser. 58, AGU, p. 343

Gosling, J.T. and Birn, J. 1995, Geophys. Res. Lett., 22, 869

Hagyard, M. J., Smith, J.R., Jr., Teuber, D. and West, E.A. 1984, Solar Phys., 91,115

Hagyard, M., Venkatakrishnan, P. and Smith, J.B., Jr. 1990, ApJS, 73, 159

Hale, G.E. 1927, Nature 119, 708

Hale, G.E. 1925, Mt. W. Comm. No. 95, Proc. Nat. Acad. Sci. II, p. 691

Harvey, J.W. 1994, BAAS, 26, 1523

Klein, L.W. and Burlaga, L.F. 1982 J. Geophys. Res., 87, 613

Leroy, J.-L. 1978, A\&A, 64, 247

Leroy, J.-L. 1989, in Dynamics and Structure of Quiescent Solar Prominences,

(ed.) E.R. Priest, Kluwer Acad. Publ., Dordrecht, Holland, p. 77

Low, B.C. and Hundhausen, A. 1995, ApJ, 443, 818

Martin, S.F. 1994, BAAS, 26, 1522 
Martin, S.F. and Echols, C.R. 1994, in Solar Surface Magnetism, (eds.) R.J. Rutten and C.J. Schrijver, Kluwer Acad. Publ., Dordrecht, Holland, p. 339

Martin, S.F., Bilimoria, R. and Tracadas, P.W. 1994, in Solar Surface Magnetism, (eds.) R.J. Rutten and C.J. Schrijver, Kluwer Acad. Publ., Dordrecht, Holland, p. 303

Martin, S.F. and McAllister, A.H. 1995a, BAAS, 27, 961

Martin, S.F. and McAllister, A.H. 1995b, in Magnetodynamic Phenomena in the Solar Atmosphere, (eds.) Y. Uchida et al., Kluwer Acad. Publ., Dordrecht, Holland, p. 497

Martin, S.F. and McAllister, A.H. 1997, in Coronal Mass Ejections, (eds.) N. Crooker et al., Geophys. Mono. Ser. 99, AGU, p. 127

Martin, S.F. and McAllister, A.H. 1998, ApJ., submitted

Marubashi, K. 1986, Adv. Space Res., 6, 335

Marubashi, K. 1997, in Coronal Mass Ejections, (eds.) N. Crooker, J.A. Joselyn and J. Feynman, Geophys. Mono. Ser. 99, AGU, p. 147

Pevtsov, A.A., and Canfield, R.C. 1997, ApJL, submitted

Pevtsov, A.A., Canfield, R.C., and Metcalf, T.R. 1994, ApJL, 425, L117

Richardson, R.S. 1941, ApJ, 41, 24

Rompolt, B. 1975, Acta Univ. Wratenslavensis No. 252, 55

Rompolt, B. 1990, Hvar Obs. Bull. 14(1), 37

Rust, D.M. 1994, Geophys. Res. Lett. 21, 241

Rust, D.M. and Bar, V. 1973, Solar Phys., 33, 445

Rust, D.M. and Kumar, A. 1994, Solar Phys., 155, 69

Rust, D.M. and Kumar, A. 1996, ApJ, 464, L199

Rust, D.M. and Martin, S.F. 1994, in Solar Active Region Evolution, (eds.) K.S. Balasubramaniam and G.W. Simon, ASP Conf. Ser., Vol. 68, San Francisco, p. 337

Seehafer, N. 1990, Solar Phys., 125, 219

Zhang, H. 1990, The Solar Cycle, (ed.) K.L. Harvey, ASP Conf. Ser., Vol. 27, San Francisco, p. 124

Zirker, J.B., Martin, S.F., Harvey, K., and Gaizauskas, V. 1997, Solar Phys., 175,27 